\title{
Labor
}

\section{Die Herausgeber}

In der Labor-Rubrik der LiLi erscheinen - unabhängig von den thematischen Schwerpunkten, denen üblicherweise die einzelnen Hefte gewidmet sind - Texte zu wissenschaftlich, literarisch oder kulturell-gesellschaftlich aktuellen Themen, innovative Versuche (der Form oder dem Inhalt nach) oder Beiträge zu Fragen, die es verdienen, kontinuierlich gestellt und bearbeitet $\mathrm{zu}$ werden.

In den letzten Jahren und Monaten sind viele Autorinnen und Autoren der Einladung der Herausgeber gefolgt, für dieses offene Forum Beiträge einzureichen, und eine recht große Zahl von Texten wurde nach einem externen und internen Begutachtungs- und z. T. Überarbeitungsprozess schließlich zur Veröffentlichung angenommen. Da in dem gleichen Zeitraum einige Thementeile so umfangreich ausfielen, dass in den entsprechenden Heften keine Labor-Beiträge mehr erscheinen konnten, warten die Autorinnen und Autoren zum Teil bereits seit längerer Zeit auf die Veröffentlichung dieser Texte.

Um die Artikel dem Publikum nicht länger vorzuenthalten, haben sich die Herausgeber daher dazu entschlossen, wie schon einmal, im Fall von Heft 112, eine Ausgabe vorzusehen, die ausschließlich aktuelle Labor-Beiträge bringt. Wie damals ergibt sich so insgesamt ein „Kaleidoskop“, das den Leser, die Leserin dazu einlädt, die Grenzen zwischen den Teildisziplinen als bewegliche wahrzunehmen und sich durch die Formen und Farben, in denen sich die Germanistik und ihre Nachbarfächer heute präsentieren, vielfältig anregen zu lassen.

Prof. Dr. Hartmut Bleumer, Universität Göttingen

Prof. Dr. Rita Franceschini, Freie Universität Bozen/Libera Università di Bolzano

Prof. Dr. Stephan Habscheid, Universität Siegen

Prof. Dr. Niels Werber, Universität Siegen 